viel ein alter ausgewachsener Strauss wiegen möge. Es fanden sich hierüber die verschiedensten sich arg widersprechenden Angaben bei den einzelnen Autoren. Mit Rücksicht auf diese Frage theilt Herr Re i c h e n o w eine ihm von Herrn Dr. B o l a u in Hamburg übersandte Notiz mit. Dieselbe lautet:

Ich hatte ganz vor kurzem Gelegenheit, einen sehr grossen und ausserordentiich fetten männlichen Strauss, Struthio camelus, zu wiegen und fand das Gewicht des Gadavers 170 Pfund. Die brauchbaren Federn (zum Schmuck) wogen 975 Gramm; nehmen wir an, dass ebenso viele, deren Gewicht ich nicht feststellte, beseitigt wurden, so würde sich das Gesammtgewicht auf 174 Pfund berechnen; ein höheres dürfte selten oder nie gefunden werden."

Schlnss der Sitzung.

Schalow.

Cabanis, Gen.-Secr.

\title{
Bericht über die April-Sitznng.
}

Verhandelt Berlin, Montag den 7. April 1884, Abends 8 Uhr im Sitzungslocal.

Anwesend die Herren: Cabanis, Reichenow, Grunack, Thiele, v. Dallwitz, Schalow, Mützel, Matschie, Krüger-VeIthusen und Thienen.

Als Gäste die Herren: Lackowitz sen., Lackowitz jun. und Spiess (Charlottenburg).

Vorsitzender: Herr Cabanis. Schrifte: Herr Matschie.

Nach Verlesung des Berichtes über die März-Sitzung nimmt Herr $\mathrm{Cab}$ an is das Wort zur Besprechung einiger im Laufe des verflossenen Monats erschienenen Publicationen. Er theilt mit, dass der im Auftrage der Gesellschaft im Jahre 1875 von Herrn Reichenow erlassene Aufruf zur Begründung ornithologiseher Beobachtungsstationen Deutschlands (mit Einschluss von Oesterreich und der Schweiz) in den verschiedensten Ländern Anregung zur allgemeineren wissenschaftlichen Beobachtung der Vögel gegeben habe. Herrn Dr. R. Blasius, der nach Reichenow die Redaction der Jahresberichte übernommen hat, ist es gelungen, in Oesterreich leitende Kreise für das Unternehmen zu interessiren, so dass jetzt als abgezweigte selbstständige Publication der erste Jahresbericht (1882) des Comités für ornithologische Beobachtungs-Stationen in Oesterreich und Ungarn, welcher 347 Arten als beobachtet aufführt, in der Stärke von 
200 Seiten vorliegt. In Ungarn beginnt sich ebenfalls eine regere Thätigkeit für die Zwecke unserer Wissensehaft bemerklieh zu machen, welche zur Begründung der von Dr. Julius v. Madarász herausgegebenen "Zeitschrift für die gesammte Ornithologie" geführt hat. England ist in allerneuster Zeit mit Beobachtungsstationen versehen worden und in Amerika hat sich im September 1883 in New-York ein Comite $\theta$ on Migration of B ir ds gebildet, dessen Aufruf sich in der ersten Nummer eines neu gegründeten Journals: The Auk: A Quarterly Journal of Ornithology, findet. Diese Zeitschrift, welche die Fortsetzung des "Bulletin of the Nuttal Ornithological Club" bildet, verspricht unter Redaction der Herren J. A. Allen, Elli ot Coues, Ridgway, Brewster und $\mathrm{Chamberlain}$ eine werthvolle Bereicherung unserer periodischen Litteratur zu werden. Aus der Reihe der Publicationen, welche, wie die erwähnten, den Anwesenden vorgelegt wurden und durch die Herren Cabanis, Reichenow und $\mathrm{Sch}$ alow eine mehr oder weniger eingehende Besprechung erfuhren, seien noch folgende erwäbnt: Ka lender für $G$ efl ù gelfreunde: redigirt von Gustav Meyer. - Die April-Nummer des Ibis mit einem Beitrag Seebohm's zur Japanischen Fauna. - Eine Arbeit unseres auswärtigen Mitgliedes Prof. Lie be über: Unsere Taucher. - Joseph Talsky: Ueber das Vorkommen und die Erbeutung von Adlerarten in Mähren.

Hierauf erhält Herr R eich enow das Wort zu einem Vortrage: Zur Geschichte der ornithologischen Systematik, welcher kurz die hauptsächlichsten Systeme seit der Mitte des vorigen Jabrhunderts behandelte. Der Gegenstand wird eine ausführlichere Betrachtung in der 38. Lieferung der Encyklopädie der Naturwissenschaften erfahren. Dr. Reichenow theilte alsdann mit, dass sich unter den von Dr. Böhm eingesendeten Vögeln wiederum eine neue Art gefunden habe; für welche er den Namen Bradyornis Böhmi Rchw.

vorschlägt. Oberkopf und Rücken fahlbraun mit dunkelbraunen Längsstrichen; auf dem Vorderkopfe und im Nacken ist die Grundfarbe lichter, bräunlich weiss; Bürzel blass rostbraun; Kopfseiten und ganze Unterseite weiss, auf Kopfseiten, den Seiten der Kehle, Kropf, Brust und Weichen schwarz gefleckt oder gestrichelt, Weichen bräunlich verwaschen; Schwingen, deren grosse Deckfedern und Schwanzfedern dunkelbraun mit rostfarbenen Aussensäumen, Schwingen mit fahlbräunlichen Innensäumen. Länge ca. 130, 
Fliggel 77, Schwanz 48, Firste 12, Lauf $17 \mathrm{Mm}$. - Fundort: Kakomá. - Hinsichtlich der Flügelbildung und Sehnabelform stimmt die Art mit den typischen Formen der Gattung Bradyornis überein. Die Färbung ist freilich eine sehr abweichende.

Der Vorsitzende, Herr $\mathrm{Cab}$ anis, legt hierauf einen Pieper vor, der von der Deutschen Südpolarexpedition in Süd-Georgien durch Dr. v. Steinen gesammelt ist und den Herr Cabanis als Anthus antarcticus n. sp.

charakterisirt. Dieser Vogel, den die Expedition zusammen mit Chionis alba Quoi et G. und Querquedula Eatoni Sharpe als einzige Landvögel fand, hat die Grösse einer Feldlerche und ist somit einer der grössten Pieper. Er ist durch seine Grösse, durch die starken Tarsen und langen Zehen sowie durch die stärker markirten längeren dreieckigen Schafttlecke an der Brust und den Weichen auf den ersten Blick von seinen nächsten Verwandten, A. correndera Vieill., furcatus Orb. und bogotensis Sel. zu unterscheiden. Die äusserste Steuerfeder ist jederscits an der Aussenfahne und längs des Schaftes der Innenfahne weiss. Der Spitzentheil derselben an der Aussenfahne dunkel, an der Innenfahne weiss. Alle übrigen Steuerfedern ohne weisse Färbung. Unterschwanzdecken an der Basalbälfte sowie ein Schaftstrich an der Spitze derselben dunkelbraun. Lg. 180, al. 86, caud. 73, rostr. 15, tars. 23, hall. cum ungue 24, ung. 12, dig. med. cum ungue 23. Das Exemplar ist als $\delta$ bezeichnet. Hab.: Suid-Georgien. [Nach einen Bericht der Expedition ist er in Süd-Georgien nicht selten, unter dem Schnee in den Büscheln des Tussackgrases nach Käfern suchend.]

Schluss der Sitzung. Matschie. $\mathrm{Ca} b \mathrm{a}$ is, Gen.-Secr. 